\title{
Rotor Unbalance Kind and Severity Identification by Current Signature Analysis with Adaptative Update to Multiclass Machine Learning Algorithms
}

\author{
H. M. Schaberle ${ }^{1}$, S. Youssef ${ }^{1}$, F. S. Pacheco ${ }^{1}$, C. A. Penz ${ }^{1}$, \& S. L. Ávila ${ }^{1}$ \\ ${ }^{1}$ Electrical and Electronic Engineering Department, Federal Institute of Santa Catarina, 950, Av. Mauro Ramos, Centro, \\ 88034-495, Florianópolis, Santa Catarina, Brazil
}

Correspondence: S. L. Ávila, Electrical and Electronic Engineering Department, Federal Institute of Santa Catarina, 950, Av. Mauro Ramos, Centro, 88034-495, Florianópolis, Santa Catarina, Brazil.

Received: April 15, $2021 \quad$ Accepted: April 30, $2021 \quad$ Online Published: May 19, 2021

doi:10.11114/set.v8i1.5213 URL: https://doi.org/10.11114/set.v8i1.5213

\begin{abstract}
The health of a rotating electric machine can be evaluated by monitoring electrical and mechanical parameters. As more information is available, it easier can become the diagnosis of the machine operational condition. We built a laboratory test bench to study rotor unbalance issues according to ISO standards. Using the electric stator current harmonic analysis, this paper presents a comparison study among Support-Vector Machines, Decision Tree classifies, and One-vs-One strategy to identify rotor unbalance kind and severity problem - a nonlinear multiclass task. Moreover, we propose a methodology to update the classifier for dealing better with changes produced by environmental variations and natural machinery usage. The adaptative update means to update the training data set with an amount of recent data, saving the entire original historical data. It is relevant for engineering maintenance. Our results show that the current signature analysis is appropriate to identify the type and severity of the rotor unbalance problem. Moreover, we show that machine learning techniques can be effective for an industrial application.
\end{abstract}

Keywords: rotating electric machines, current signature analysis, rotor unbalance, diagnostics, machine learning

\section{Introduction}

Industrial applications demand conversion of energy from electrical to mechanical type. The global rotating electrical machine market is estimated at \$ 36 billion by 2024 (Global Market Insights, 2019). Maintaining the optimal condition of those machines and extending their useful life to the maximum is essential to guarantee the return of investments and profitability for companies.

Fault diagnosis in addition to condition monitoring instrumentation improves safety and reliability in rotating electrical machine operation (Toliyat et. al 2017). It also supplies a solid foundation in the fundamentals of fault cause and effect. Most of the existing techniques for condition monitoring and fault diagnosis are based on measurements of the vibration (Finley et. al, 2015), temperature (Hey et. al, 2016), current signature (Thomson and Culbert, 2017), or external electromagnetic field (Rigoni et. al, 2017). The goal of these different techniques is a search for accurate and reliable methodologies.

The motor current signature analysis (MCSA) has been used for condition monitoring in industrial applications. MCSA typically uses frequency domain analysis of the current signal through steady-state machine operation. An advantage of MCSA over other techniques is the simplicity of its instrumentation as presented in Section 3. There is no requirement for complex mechanical adjustments like the ones needed for vibration sensors (accelerometers and proximeters). Nevertheless, interferences and noise can hinder the MCSA.

One recent work regarding MCSA for unbalance and misalignment is proposed by (Antonino-Daviu and Popaleny, 2018). They propose a short-time Fourier transform (STFT) approach to detect problems explicitly due to mistaken installation of induction motors. Here, we look for a different circumstance: in the regular use of any machinery, deterioration of alignment and balancing is expected. Monitoring the unbalance condition is critical for planning 
maintenance stops, and finding the most cost-effective point in time is one of the major concerns for engineering.

Still, about MCSA, different signal processing techniques are presented in the literature. Chen et. al (2016) proposes a review of wavelet transform (WT) for fault diagnosis of rotating machinery, gearbox fault cases. Another signal processing technique is empirical mode decomposition (EMD). Feng et. al (2017) review EMD methods and their applications for machinery fault diagnosis. Both studies state that WT, EMD, and data-driven threshold shrinkage help to enhance the diagnosis effectiveness. However, these techniques are based on an iterative process including the operations of interpolations and sifting. Therefore, they are user-dependent algorithms.

The chief benefit of end-to-end machine learning (ML) procedures is that less domain expertise is required to achieve good results. One review is Khan and Yairi (2018). They discuss the application of ML techniques to system health management in machinery overall sense. They point out strengths and restrictions of supervised learning (decision trees (DTC), one-vs-one (OvO), random forest, naïve Bayes, Bayesian networks, support vector machines (SVM) and multilayer perceptrons), unsupervised learning (k-means, adaptive resonance theory, hidden Markov models), and more complex algorithms like an autoencoder, convolutional neural networks, and recurrent neural networks. They conclude that despite improvements misclassifications are always possible, and additional sensors may be considered (MCSA, vibration, electromagnetic fields, and thermic variations put all together).

We agree that the more information one has access to, the easier the system health management. Despite that, unlike controlled laboratory environments, industrial locations may hinder the setting up of the sensors in the best place and under the best conditions.

In this context, we propose this paper. For a relevant real-world industrial issue (rotor unbalance due to natural aging), with a low-priced, simple, and easy to install instrumentation (MCSA), we propose an effective adaptative training nonlinear multiclass SVM, DTC, and OvO.

ML multiclass classification is identifying occurrences into one of three or more classes (Liu, 2019). Rotor unbalance kind and severity classification is a multiclass problem. There are four classes of unbalanced failure with severity indexes, according to ISO 1940-1. We have three severity levels for each class analysis. The section 2 explains the rotor unbalance problem. Multiclass classification makes the statement that each sample is assigned to only a single class.

From time to time any machinery has to be stopped for maintenance (either predictive, preventive, or corrective). When the equipment returns to operational status, one can obtain the healthy condition signature. Our initial data training set is assembled with part of the healthy signal and part of every possible failure (4 kinds and 3 severities). A supervised ML algorithm takes a labeled set of input datasets and its familiar responses (healthy condition signature) to learn the classification model (Liu, 2019). Thus, a supervised learning procedure is recommended.

Among the supervised learning techniques, SVM, OvO, and DTC emerge because they can efficiently perform a multiclass and nonlinear classification (Hussain, 2019). It is nonlinear due to the disparity of the motor conditions operation, for example, power supply variations.

According to our knowledge, one of the first works using SVM for unbalance detection is proposed by Baccarini et. al (2011). Tsui et. al (2014) presented a discussion of OvO and DTC approaches for bearing faults diagnostics. Both research works use mechanical vibration instrumentation. Another related study, proposed by Matić et. al. (2012), employs MCSA and SVM for the specific application of broken rotor bar.

Our studies cover three years of observation on the laboratory test bench (2017 2020). Beyond the natural unbalances due to use and regular operation of the motor - the focus of this work - other misalignments occurred due to ambient temperature range, building vibrations, or test bench displacements for any reason. Another concern is the noise from the power source and the vector inverter motor control, which change over time.

It imposes difficulties in the classification of the current data with the training set done back in 2017. A similar issue is discussed by Chao et. al (2019). They proposed a total reconstructed training-set SVM with roulette cooperative coevolution. It is a complex probabilistic strategy and, therefore, can be less assertive. In our problem context, we choose to preserve the original training set to keep the initial condition. We understand that this is crucial for maintenance engineering. Our proposal is from time to time to add a small percentage of current data in the training set, as discussed in section 6.3 .

Therefore, the present proposal is justified: adaptative update nonlinear multiclass SVM, OvO, and DTC for rotor unbalance kind and severity classification by current signature analysis.

\section{Unbalance Rotor Problem}

Fundamentals of rotor balancing are contained in ISO 19499. ISO 21940-11 establishes procedures and unbalance tolerances for balancing rotors with rigid behavior. It specifies: a) the permissible residual unbalance magnitude; b) the necessary number of correction planes; c) the allocation of the permissible residual unbalance to the tolerance planes, 
and d) how to account for errors in the balancing process.

This issue is characterized by the existence of mass unbalanced on the rotor. In this condition, the center of mass does not coincide with the axis of rotation. Each lack of symmetry causes a displacement in the center of gravity; the sum of these displacements results in the deviation of the center of mass relative to the axis of rotation. Unbalance is measured in units as g.mm and is equal to the product of the unbalance mass by the distance from the axis of rotation to the resulting mass center (ISO 21940-14).

There are four types of unbalance (Matsushita, 2017): static, quasi-static, coupled, and dynamic. This classification considers the relative position between the axis of rotation and mass. Figure 1 shows the types of unbalanced rotors.
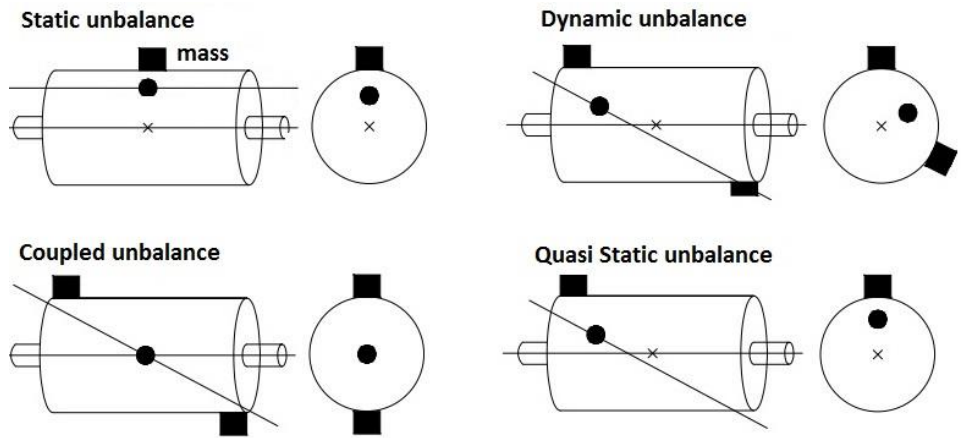

Figure 1. Different kinds of rotor unbalanced failure

Static unbalance occurs when the mass axis is displaced parallel to the axis of rotation. It is called static because even when the machine is turned off, the axis will rotate until the point of greatest mass is down, making it detectable.

In the coupled unbalance the mass axis intercepts the axis of rotation in the center of gravity of the rotor. This unbalance is equivalent to two masses in two different planes, displaced $180^{\circ}$ each other. The amplitude of this unbalance can no longer be determined simply by mass and radius, since it is also necessary to define the distance between the equivalent masses, since both masses exert a moment on the axis.

Dynamic unbalance occurs when the mass axis is not parallel to and does not intercept the axis of rotation. It is common for a series of small unbalances to occur that can be defined by equivalent dynamic unbalance. This unbalance can be represented by the combination of equivalent static and coupled unbalance.

In quasi-static unbalance, the mass axis intercepts the axis of rotation at a point other than the center of gravity. This unbalance is considered a special case of dynamic unbalance (Matsushita, 2017). Therefore, quasi-static unbalance classification can be not considered.

\subsection{Rotor Unbalance Severity Levels}

Severity levels of the fault under study were based on ISO 1940-1 balance quality grades (G) for electric motors and generators with a shaft height of at least $80 \mathrm{~mm}$. For maximum operating speed of up to $950 \mathrm{rpm}$, the standard recommends grade G 6.3. Above $950 \mathrm{rpm}$, grade G 2.5 is recommended.

As cited before, a laboratory test bench was built according to ISO's standards quoted. Through the mechanical design of the bench shaft, its measurements and mass estimation were obtained as disc diameter is $250 \mathrm{~mm}$; total shaft length is $760 \mathrm{~mm}$; distance between disks is $166.2 \mathrm{~mm}$; mass estimate of $38.78 \mathrm{~kg}$. Figure 2 shows the motor, shaft, and discs used to cause unbalance issues. Table 1 shows the maximum unbalance modulus as a function of the motor feed frequency and the quoted balance quality notes calculated according to ISO 1940-1.

Table 1. Recommended maximum misalignments per ISO 1940-1 due experimental tests bench

\begin{tabular}{cccc}
\hline \multirow{2}{*}{ Frequencies $(\mathrm{Hz})$} & $\begin{array}{c}\text { Synchronous Speed } \\
(\mathrm{RPM})\end{array}$ & $\begin{array}{c}\text { Grade } \\
\text { G } 6.3\end{array}$ & $\begin{array}{c}\text { Grade } \\
\text { G 2.5 } \\
(\mathrm{g}) \mathrm{mm})\end{array}$ \\
\hline 5 & 150 & 15553.84 & 6172.16 \\
7,5 & 225 & 10369.22 & 4114.77 \\
10 & 300 & 7776.92 & 3086.08 \\
\hline
\end{tabular}

Based on these values, three levels of severity were established for the tests. Table 2 presents these levels with the positioning of the unbalance masses on the disks and the unbalance vector data in the planes. Disc 1 was considered to be the disc closest to the motor, disc 2 the central disc, and disc 3 is the disc of the opposite end, as shown in Figure 2. The calculation plans are: plane 1 positioned on disk 1 and plane 2 positioned on disk 3 . Table 2 shows that we are able to emulate all recommended unbalanced limits established per ISO 1940-1. The red circles in Figure 2 highlight the coupled unbalance failure with severity level 3 , according to Table 2 . 
Table 2. Severity of the unbalances generated

\begin{tabular}{|c|c|c|c|c|c|}
\hline \multicolumn{6}{|c|}{ Mass Positioning: level 1} \\
\hline Unbalances & Disc $1(\mathrm{~g})$ & $\operatorname{Disc} 2(g)$ & $\operatorname{Disc} 3(\mathrm{~g})$ & $\begin{array}{l}\text { Plan } 1 \\
\text { (g.mm) }\end{array}$ & $\begin{array}{l}\text { Plan } 2 \\
\text { (g.mm) }\end{array}$ \\
\hline Static & - & $18.1 / 0^{\circ}$ & - & $2018.7 / 0^{\circ}$ & $2018.7 / 0^{\circ}$ \\
\hline Coupled & $18.1 / 0^{\circ}$ & - & $18.1 / 180^{\circ}$ & $2018.7 /-90^{\circ}$ & $2018.7 / 90^{\circ}$ \\
\hline Dynamic & $18.1 / 0^{\circ}$ & - & $18.1 / 90^{\circ}$ & $2018.7 / 0^{\circ}$ & $2018.7 / 90^{\circ}$ \\
\hline \multicolumn{6}{|c|}{ Mass Positioning: level 2} \\
\hline Unbalances & Disc $1(\mathrm{~g})$ & Disc $2(g)$ & $\operatorname{Disc} 3(\mathrm{~g})$ & $\begin{array}{l}\text { Plan } 1 \\
\text { (g.mm) }\end{array}$ & $\begin{array}{l}\text { Plan } 2 \\
\text { (g.mm) }\end{array}$ \\
\hline Static & - & $35.7 / 0^{\circ}$ & - & $4159.1 / 0^{\circ}$ & $4159.1 / 0^{\circ}$ \\
\hline Coupled & $35.7 / 0^{\circ}$ & - & $35.7 / 180^{\circ}$ & $4159.1 /-90^{\circ}$ & $4159.1 / 90^{\circ}$ \\
\hline Dynamic & $35.7 / 0^{\circ}$ & - & $35.7 / 90^{\circ}$ & $4159.1 / 0^{\circ}$ & $4159.1 / 90^{\circ}$ \\
\hline \multicolumn{6}{|c|}{ Mass Positioning: level 3} \\
\hline Unbalances & Disc $1(\mathrm{~g})$ & $\operatorname{Disc} 2(g)$ & $\operatorname{Disc} 3(\mathrm{~g})$ & $\begin{array}{l}\text { Plan } 1 \\
\text { (g.mm) }\end{array}$ & $\begin{array}{l}\text { Plan } 2 \\
\text { (g.mm) }\end{array}$ \\
\hline Static & - & $65.3 / 0^{\circ}$ & - & $7607.4 / 0^{\circ}$ & $7607.4 / 0^{\circ}$ \\
\hline Coupled & $65.3 / 0^{\circ}$ & - & $65.3 / 180^{\circ}$ & $7607.4 /-90^{\circ}$ & $7607.4 / 90^{\circ}$ \\
\hline Dynamic & $65.3 / 0^{\circ}$ & - & $65.3 / 90^{\circ}$ & $7607.4 / 0^{\circ}$ & $7607.4 / 90^{\circ}$ \\
\hline
\end{tabular}

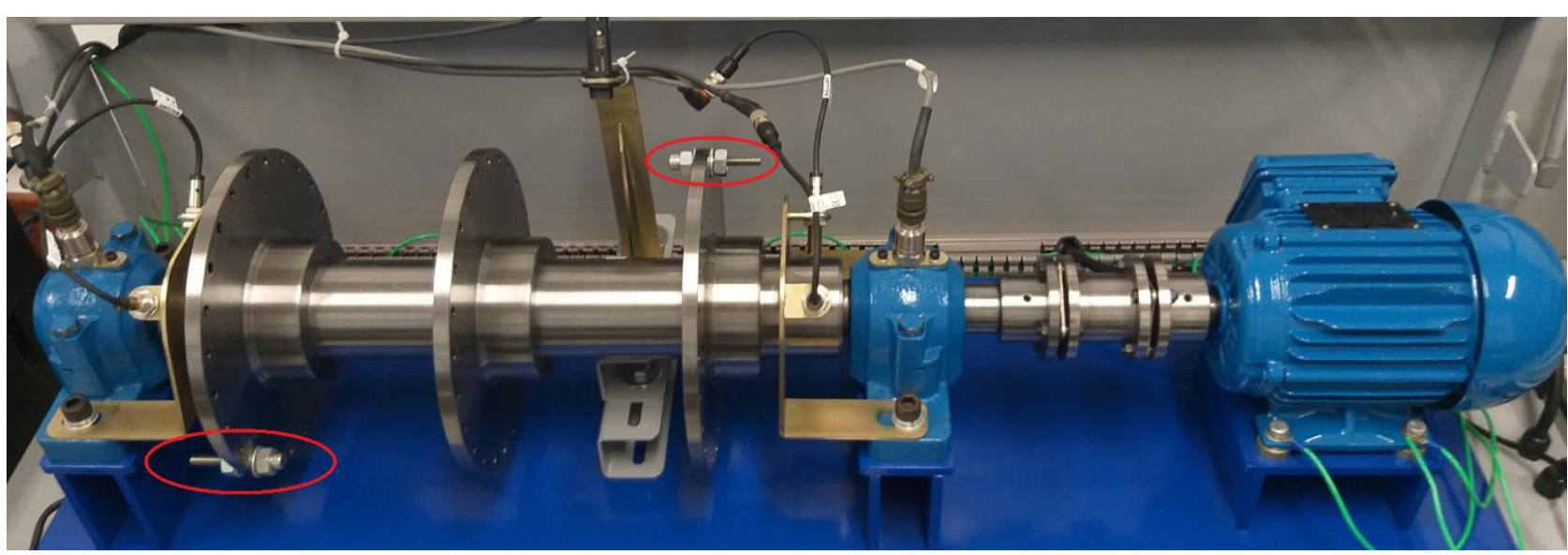

Figure 2. Laboratorial test bench - coupled unbalance, level 3 (red circles)

\section{The Stator Current Sensing}

The selected sensor for the current measurements was the LA 25-NP. The LA 25-NP is a Hall effect current transducer, with galvanic isolation. It has a maximum error of $\pm 0.5 \%$ in $25^{\circ} \mathrm{C}$, response time to $90 \%$ of the nominal current $<1 \mu \mathrm{s}$ and has a variable measuring range depending on the connection of its terminals, ranging from 5 to $25 \mathrm{~A}$.

The system for acquisition and signal processing are chosen was NI USB-6212 data acquisition board. This equipment is a USB-powered multifunctional data acquisition module optimized for accuracy with high sampling rates. The module has a 16-bit resolution, sampling rate up to $400 \mathrm{kS} / \mathrm{s}$.

Figure 3 shows the current measurements at one winding of a three-phase induction motor (1 HP). Here, the measurements are done in healthy motor conditions, i.e. a balanced structure. This is a healthy current signal signature for $10 \mathrm{~Hz}$ power frequency obtained through FFT (magnitude). $10 \mathrm{~Hz}$ power frequency is due to ISO 1940-1, as shown in Table 1. We did the same procedures for one, two, and three phases alone. The analyses are similar.

\section{FFT and Hilbert for Unbalance Classification}

The frequencies in which changes were detected during the unbalance tests are close to the fundamental frequency. In this context, associating FFT and Hilbert Transform entails a better analysis of the effective value in these frequencies 
without the amplitude component influence (Rahman and Uddin, 2017) (Shrivastava and Mohanty, 2020).

Figure 4 shows the histograms obtained from the associated FFT-Hilbert Transform signals. Similar behavior is observed for (a) static and (b) dynamic unbalances, allowing kind and severity classification by many ways like a visual inspection. However, the (c) coupled unbalance kind histogram presents a bigger dispersion when compared to the others. Our observations show that dispersion increases with the unbalance severity, making diagnosis awkward by direct inspection. One possibility is working with ML techniques.

By analyzing the static unbalanced, we observed an increase in the current value at the frequency $1.5 \mathrm{f}$ when the severity level of the unbalance is greater. For instance, with the frequency of $7.5 \mathrm{~Hz}$, there was a notable increase in current values at $11.25 \mathrm{~Hz}$, as shown in Figure 5 which has the FFT magnitude of the current in the 3 severity levels.

In dynamic unbalance, we can observe a variation in current values in two frequencies. The first one is $1.5 \mathrm{f}$, the same as static unbalance, and the second one is $0.5 \mathrm{f}$. For coupled unbalanced, it was more difficult to find the frequencies that showed relevant changes, as illustrated in Figure 4 (c).

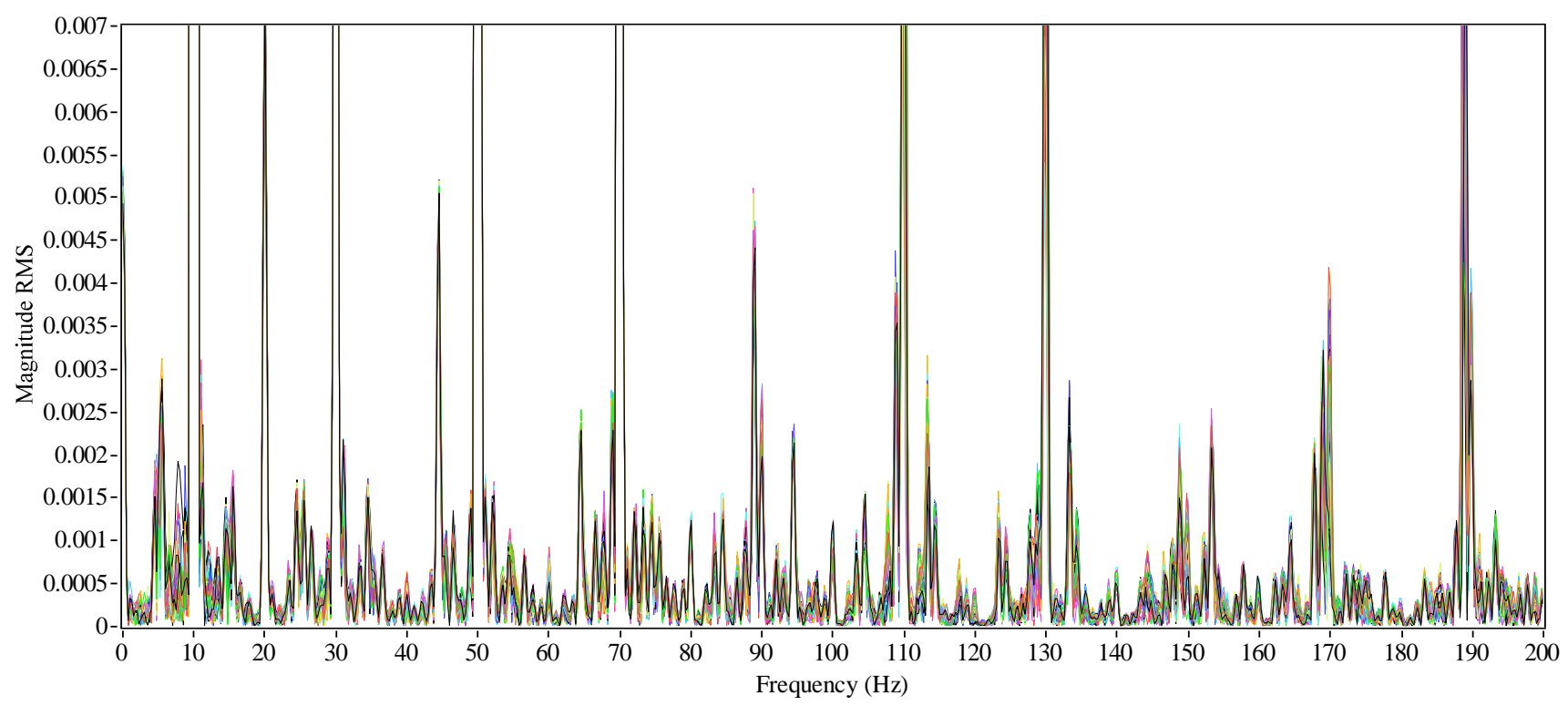

Figure 3. Current signature for healthy motor with $10 \mathrm{~Hz}$ power frequency by FFT magnitude

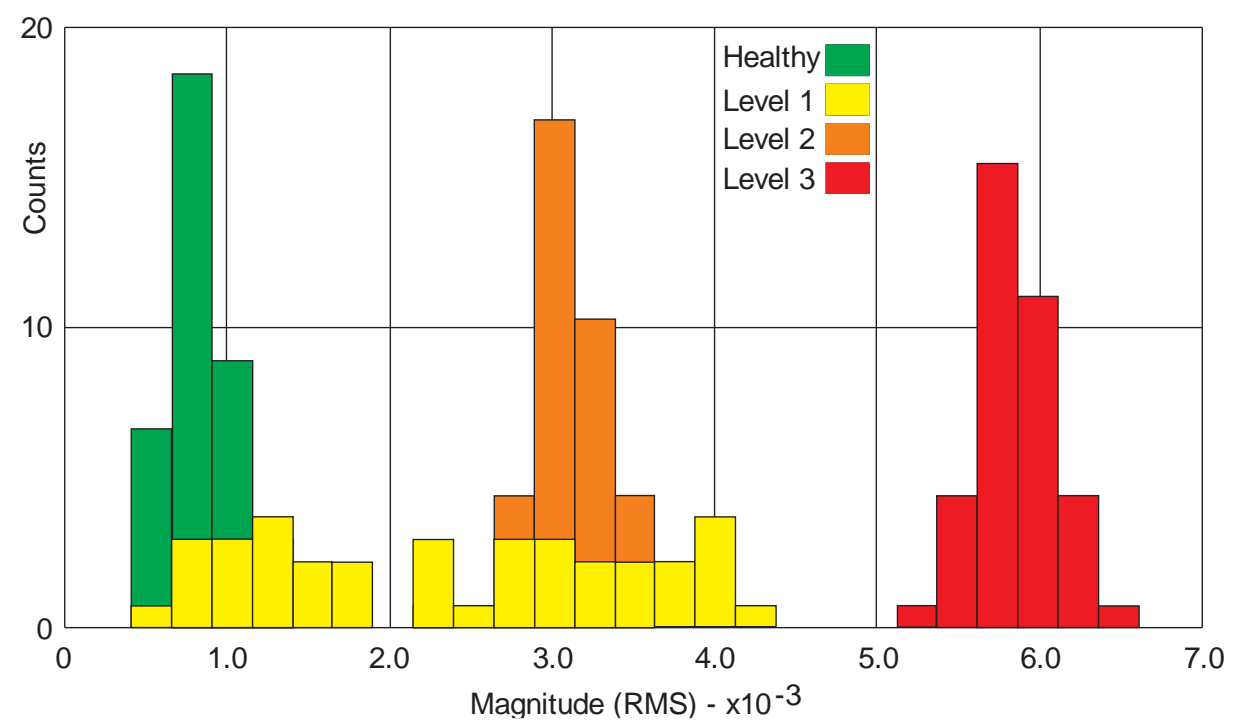

(a) Static unbalance behavior 




(b) Dynamic unbalance behavior

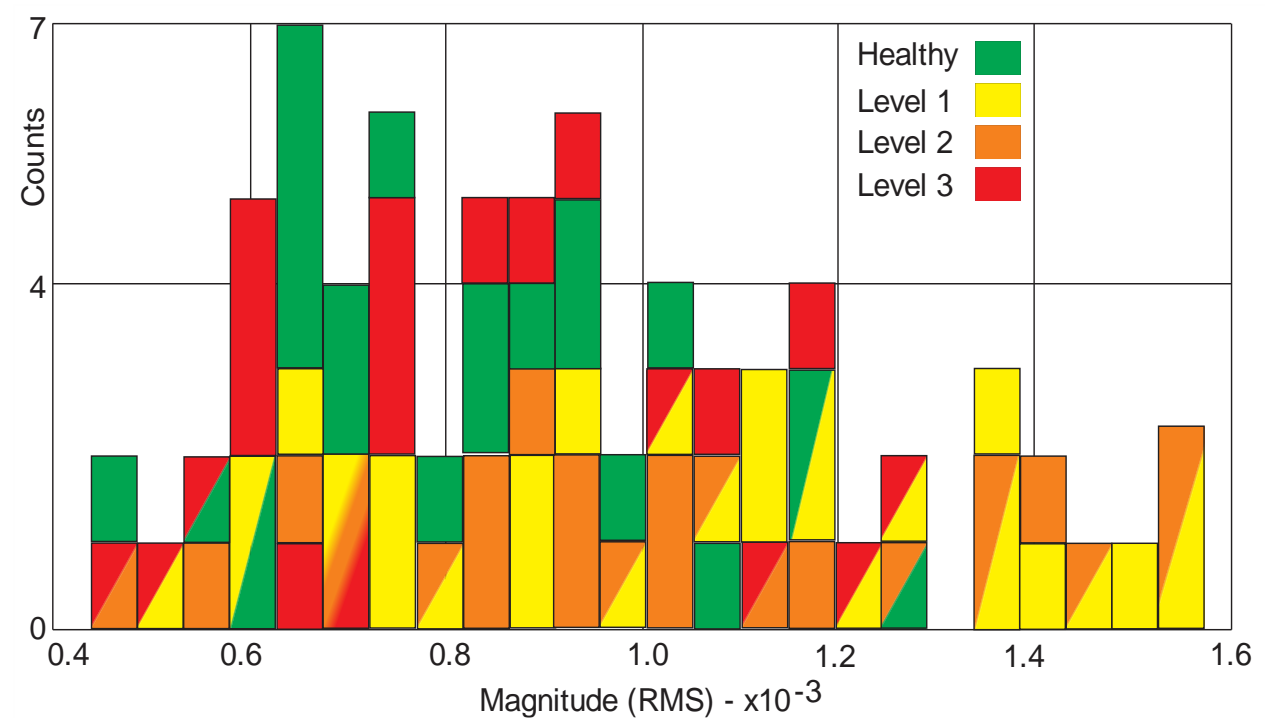

(c) Coupled unbalance behavior

Figure 4. MCSA histograms obtained from the associated FFT and Hilbert Transform signals at $0.5 \mathrm{f}(\mathrm{f}=10 \mathrm{~Hz}$ power frequency)

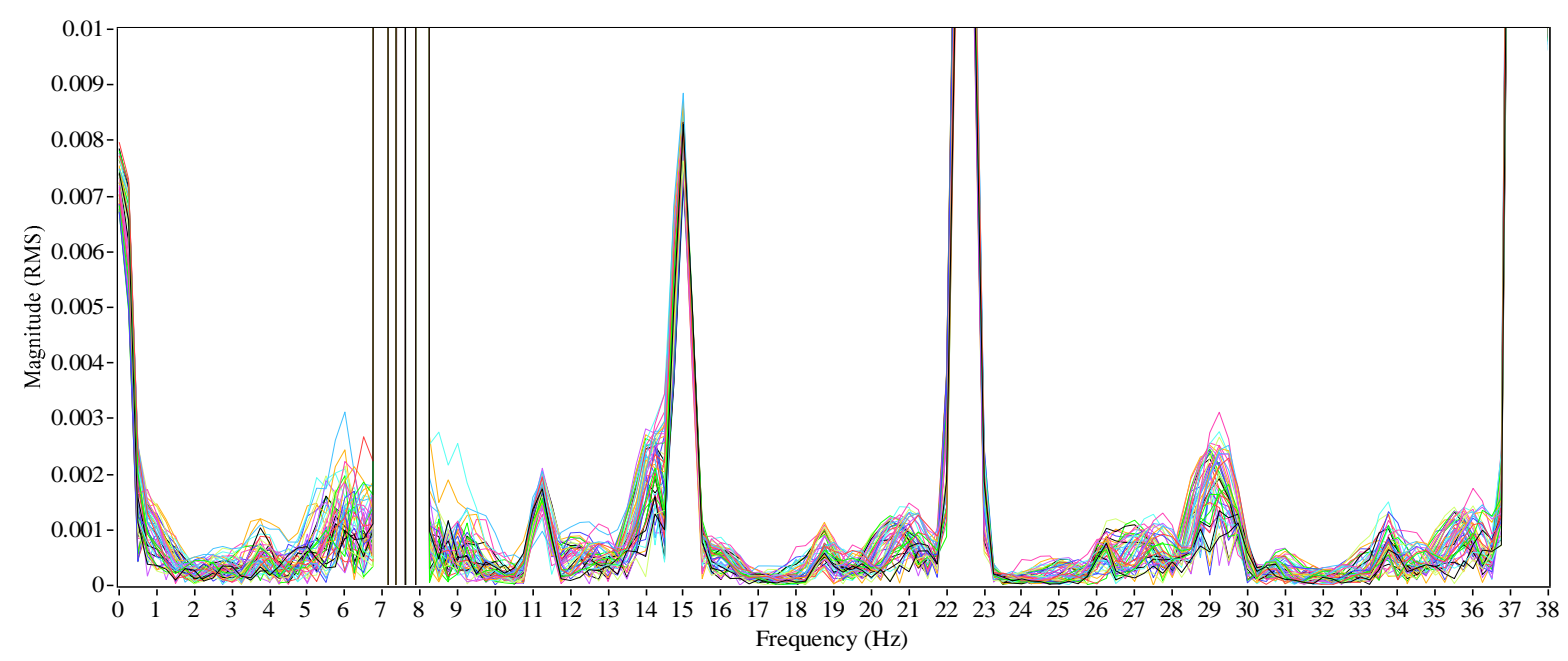

(a) Static unbalance kind at level 1 


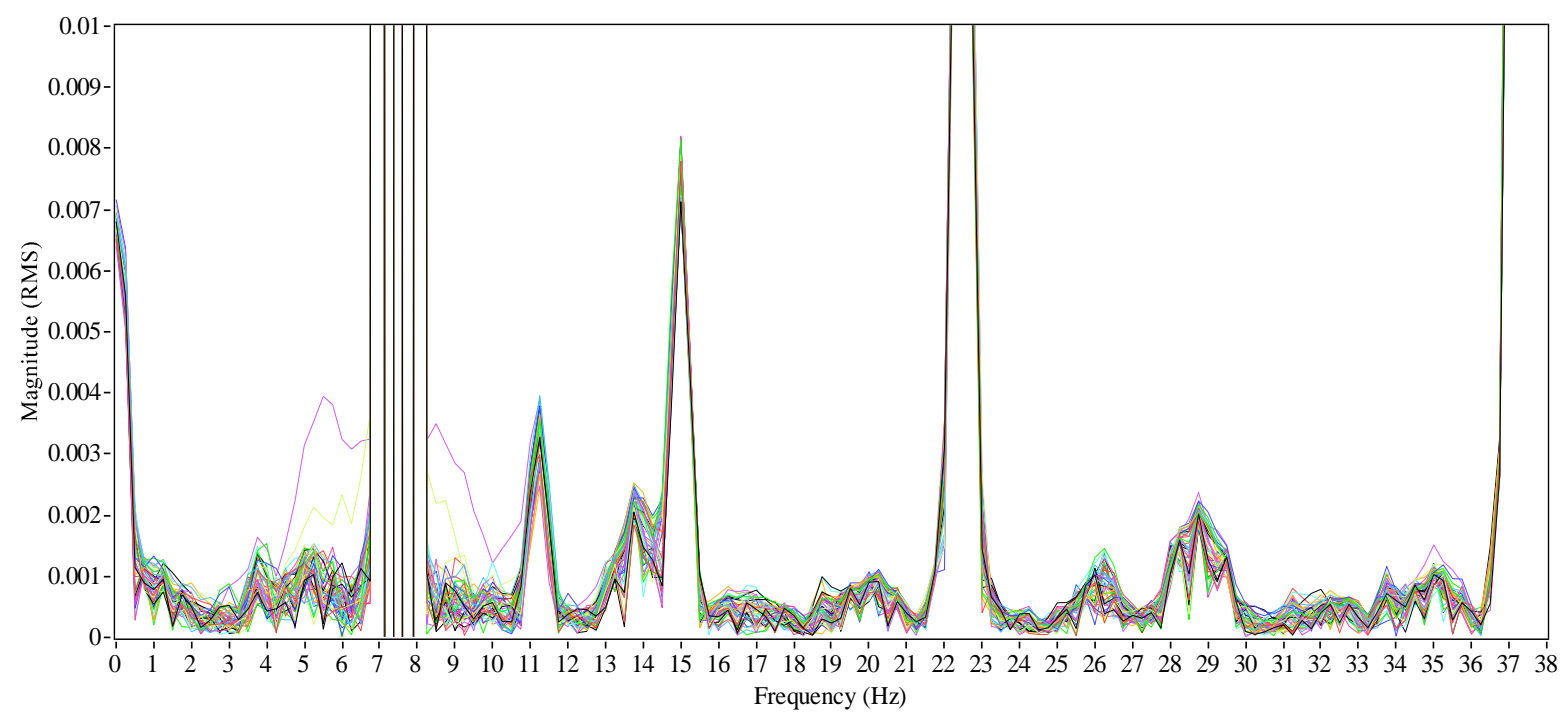

(b) Static unbalance kind at level 2

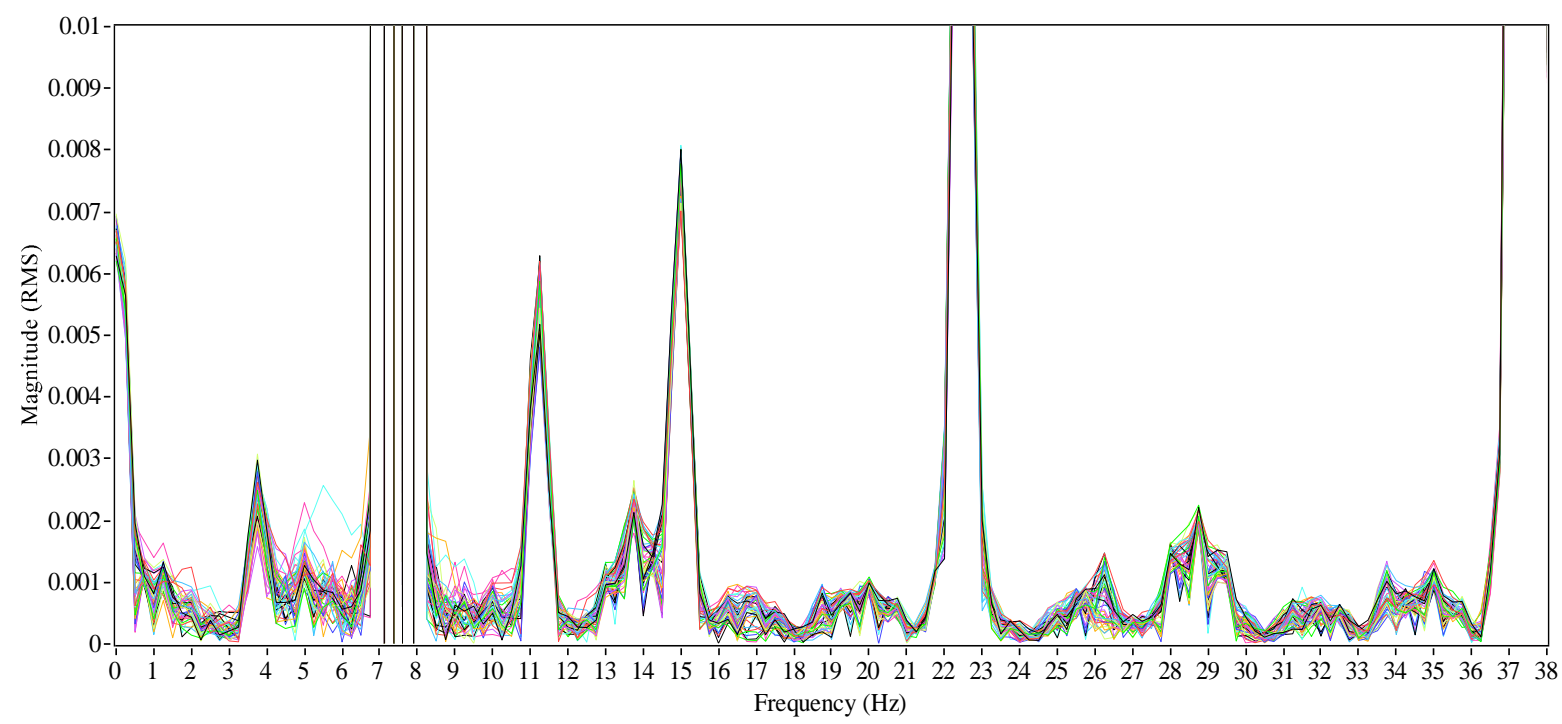

(c) Static unbalance kind at level 3

Figure 5. FFT of the stator current with fundamental frequency of $7.5 \mathrm{~Hz}$ for the motor with static unbalance kind at level 1,2 and 3

\section{Machine Learning Techniques Description}

Analyzes were performed and validated by applying three classifiers from the Python open-source library sklearn (Scikit-learn, 2020). They are SVM, DTC, and OvO classifiers (Liu, 2019). They are able to detect the unbalance rotor problem - a multiclass and nonlinear classification.

OvO procedure consists of fitting one classifier per class pair. At prediction time, the class which received the most votes is selected. This algorithm can be slower than the other similar ones, due to its $\mathrm{O}(\mathrm{n}$ _classes2) intricacy. Yet, this strategy can be suitable for algorithms that do not scale well with n_samples, like kernel algorithms, because each individual learning problem only involves a small subset of the data.

DTC assumes a split of the training data based on the values of the features to produce a generalization. The technique can indeed handle binary or multiclass classification problems. The leaf nodes can denote either one of the n_classes. One disadvantage of this classifier is the sensibility to changes in the training dataset since the splitting process can lead to very different results.

SVM maximizes the minimum distance from the separating hyperplane to the nearest example. Multiclass SVM means that additional parameters and constraints are added to the optimization problem to handle the separation of the different classes. SVM is reported as a classifier with good generalization power and robustness. One disadvantage is the lack of auditability. It is not possible to inspect why the classifier produced a result. 
We coded a script to train and test the classifiers as present in Table 3 .

Table 3. Multiclass SVM pseudo code algorithm for the training and test

- $\quad$ numpy - structures and functions to deal with matrices;

- $\quad$ lvm_read - to read .lvm files from LabVIEW;

- $\quad$ scipy.signal - to apply the STFT to the data;

- $\quad$ sklearn.metrics - to compare the ground truth with results from the classifier;

- $\quad$ sklearn.model_selection.train_test_split - to split the data set into train and test in a random means;

- sklearn.svm,

- $\quad$ sklearn.tree.DecisionTreeClassifier

- $\quad$ sklearn.multiclass.OneVsOneClassifier - the classifiers;

- $\quad$ and matplotlib.pyplot - to plot figures.

for each file in the data set:

open .lvm file - LabVIEW measurement data format

extract STFT

obtain magnitude from 0 to $200 \mathrm{~Hz}$ frequency bins

assign a label for each magnitude vector

add the magnitude vector as a line in a matrix

end for

define the fundamental frequency (f0)

define the frequencies sets

([relev_frequencies], [1.5*f0], [0.5*f0], [0.5*f0, 1.5*f0])

define the classifiers set (SVM, DTC, OvO)

for each frequency set:

extract relevant frequency bins for each line in the data

split the data set into train and test randomly

for each classifier:

train classifier

predict results for each line using the classifier

compare results using the ground truth label

end for

end for

\section{Results and Discussion}

Data acquired was exported as .lvm files. We collected 50 files for each condition in two data acquisition sessions, one in September 2017 and another in April 2019. Data from the first session served as a training set while the second session provided the test.

Lvm files, each with 200,000 samples of the acquired power current signal, were processed in the frequency domain. The function short-term Fourier transform, from the module scipy.signal with the default Hanning window, length 200,000 , and sampling frequency of $50 \mathrm{kHz}$, was the first part of the processing.

After that, we extract the magnitude in $\mathrm{dB}$ from the first 800 bins (corresponding to frequencies from 0 to $200 \mathrm{~Hz}$ ). These data are the input to the classifier. We noticed that the full magnitude spectrum from 0 to $200 \mathrm{~Hz}$ was not appropriate as input data for the classifier. Frequency bins outside the harmonics were a source of confusion for the classifier because they exhibit low energy random behavior.

The most important feature of an SVM classifier is the margin, that is, the region between two parallel hyperplanes (Hussain, 2019). Using all the bins, the margin could not be set up correctly. It is like adding noise. Besides that, we noticed that speed could be improved if we select only the relevant frequency bins.

Comparing plots from the healthy operation and static unbalance failure through a visual inspection, we can select frequency bins where the confusion between the classes was minimal. Computation tools can be used to identify which frequencies bring the most information. This way, to use the classifier we extracted the relevant frequencies from the data set, which were obtained from a minimal set with the frequencies related to the fundamental frequency. For instance, for the $5 \mathrm{~Hz}$ fundamental frequency (f), the set is formed by [4.75 55.257 .257 .57 .75$] \mathrm{Hz}$. Besides these, we also analyzed the behavior of the classifier using the following frequencies sets, [ $\left.\begin{array}{llll}1.5 & 0.5 & 1.5 \& 0.5\end{array}\right] \mathrm{f}$, where we observed a great variance of magnitude, as shown in section 4. 


\subsection{Training and Testing with 2017 Data}

The 2017 data set was randomly separated into two parts: training and test, $80 \%$ and $20 \%$ respectively. Each classifier accuracy was calculated by sklearn.metrics. Table 4(a) illustrates that a $93 \%$ accuracy average was achieved in the predictions considering the three unbalance kinds and the three severity levels by $\mathrm{OvO}$ at relevant frequency.

As happened for FFT-Hilbert Transform analysis (Figure 5(c)), the lowest hit rate was for the coupled unbalance kind (Table 4(a)). Table 4(b) presents the results obtained only for static and dynamic failure kinds. The classification rate is much better. We remember the coupled unbalance histogram presents a greater dispersion when compared to the others (Figure 5(c)). Practically and in the industrial context, this does not impose any worry. The ML classification rates found are sufficient for unbalance suspicions.

OvO, DTC and SVM have similar results in Table 4(b), excepting for SVM at relevant frequencies. Perhaps the bins were not picked appropriately, which can cause issues in identifying SVM hyperplanes. Anyway, this is not a difficulty for the OvO and DTC.

Table 5 (a) dynamic, (b) static, and (c) coupled presents the classification for the 3 levels of severity in particular. The performance of ML techniques is similar, with lighter better accuracy with OvO.

Table 4. Multiclass OVO, DTC and SVM average accuracy for the all-unbalance kinds and severity at [relevant, 0.51 .5 and $0.5 \& 1.5$ ] frequencies by training and testing with 2017 data

\begin{tabular}{|c|c|c|c|}
\hline \multicolumn{4}{|c|}{ (a) Dynamic, static and coupled unbalance } \\
\hline \multirow{2}{*}{ Frequency } & \multicolumn{3}{|c|}{ Accuracy (\%) } \\
\hline & SVM & DTC & $\mathrm{OvO}$ \\
\hline Relevant & 25 & 75 & 93 \\
\hline $1,5 \mathrm{f}$ & 55 & 52 & 54 \\
\hline $0,5 \mathrm{f}$ & 38 & 36 & 32 \\
\hline $0,5 \& 1,5 \mathrm{f}$ & 58 & 59 & 61 \\
\hline \multicolumn{4}{|c|}{ (b) Dynamic and static without coupled unbalance } \\
\hline \multirow{2}{*}{ Frequency } & \multicolumn{3}{|c|}{ Accuracy (\%) } \\
\hline & SVM & DTC & $\mathrm{OvO}$ \\
\hline Relevant & 55 & 79 & 95 \\
\hline $1,5 \mathrm{f}$ & 78 & 80 & 79 \\
\hline $0,5 \mathrm{f}$ & 58 & 57 & 55 \\
\hline $0,5 \& 1,5 \mathrm{f}$ & 83 & 85 & 82 \\
\hline
\end{tabular}

Table 5. Multiclass OVO, DTC and SVM average accuracy at [relevant $0.51 .50 .5 \& 1.5$ ] frequencies by training and testing with 2017 data for each failure kind

\begin{tabular}{|c|c|c|c|}
\hline \multicolumn{4}{|c|}{ (a) Dynamic level 1,2 and 3} \\
\hline \multirow{2}{*}{ Frequency } & \multicolumn{3}{|c|}{ Accuracy ( \%) } \\
\hline & SVM & DTC & $\mathrm{OvO}$ \\
\hline Relevant & 59 & 88 & 88 \\
\hline $1,5 \mathrm{f}$ & 95 & 94 & 95 \\
\hline $0,5 \mathrm{f}$ & 79 & 75 & 80 \\
\hline $0,5 \& 1,5 \mathrm{f}$ & 96 & 97 & 98 \\
\hline \multicolumn{4}{|c|}{ (b) Static level 1,2 and 3} \\
\hline \multirow{2}{*}{ Frequency } & \multicolumn{3}{|c|}{ Accuracy (\%) } \\
\hline & SVM & DTC & $\mathrm{OvO}$ \\
\hline Relevant & 65 & 97 & 98 \\
\hline $1,5 \mathrm{f}$ & 83 & 82 & 81 \\
\hline $0,5 \mathrm{f}$ & 79 & 79 & 80 \\
\hline $0,5 \& 1,5 \mathrm{f}$ & 97 & 97 & 98 \\
\hline \multicolumn{4}{|c|}{ (c) Coupled level 1,2 and 3} \\
\hline \multirow{2}{*}{ Frequency } & \multicolumn{3}{|c|}{ Accuracy (\%) } \\
\hline & SVM & DTC & $\mathrm{OvO}$ \\
\hline Relevant & 55 & 89 & 85 \\
\hline $1,5 \mathrm{f}$ & 37 & 35 & 45 \\
\hline $0,5 \mathrm{f}$ & 40 & 35 & 55 \\
\hline $0,5 \& 1,5 \mathrm{f}$ & 60 & 62 & 59 \\
\hline
\end{tabular}




\subsection{Training with 2017 Data and Testing with 2019 Data}

Here the training was performed with the 2017 data and testing with the 2019 data. The results were not satisfactory as the previous ones. Table 6 illustrates the poor results obtained, (a) with or (b) without coupled unbalance. It is due to the amplitude vibration involved. From 2017 to 2019, misalignments occurred due to ambient temperature range, building vibrations, or laboratory test bench displacements for any reasons that can explain the poor results here.

Table 6. Multiclass OVO, DTC and SVM average accuracy for the all-unbalance kinds and severity at [relevant, 0.5f, $1.5 \mathrm{f}$ and $0.5 \& 1.5$ ] frequencies for training 2017 and testing 2019 data

\begin{tabular}{|c|c|c|c|}
\hline \multicolumn{4}{|c|}{ (a) With coupled unbalance } \\
\hline \multirow{2}{*}{ Frequency } & \multicolumn{3}{|c|}{ Accuracy $(\%)$} \\
\hline & SVM & DTC & $\mathrm{OvO}$ \\
\hline Relevant & 17 & 16 & 17 \\
\hline $1,5 \mathrm{f}$ & 41 & 44 & 43 \\
\hline $0,5 \mathrm{f}$ & 17 & 14 & 16 \\
\hline $0,5 \& 1,5 \mathrm{f}$ & 18 & 20 & 38 \\
\hline \multicolumn{4}{|c|}{ (b) Without coupled unbalance } \\
\hline \multirow{2}{*}{ Frequency } & \multicolumn{3}{|c|}{ Accuracy $(\%)$} \\
\hline & SVM & DTC & $\mathrm{OvO}$ \\
\hline Relevant & 17 & 16 & 18 \\
\hline $1,5 \mathrm{f}$ & 44 & 43 & 43 \\
\hline $0,5 \mathrm{f}$ & 18 & 19 & 17 \\
\hline $0,5 \& 1,5 \mathrm{f}$ & 25 & 40 & 38 \\
\hline
\end{tabular}

Recently, Nalepa and Kawulok (2018) proposed a review about selecting training sets for SVM. They discussed strategies like data geometry analysis, neighborhood analysis, evolutionary methods, active learning, and random sampling methods. According to them, training ML techniques from large datasets remains an open research issue. Moreover, Chao et. al (2019) said that SVM is typically sensitive to noises present in training data. They proposed to rebuild the training set by cleaning the support vectors based on their position in high dimensional vector space. The main advantage is avoiding the effect of the fuzzy noise data.

Our rotor unbalance kind and severity classification by MCSA are not necessarily a big data problem. But it can be. It depends on the time scale used. Surely, it is noise signal data. Our main concerns are from the power source and vector inverter motor control. Besides that, misalignments occurred due to ambient temperature range, building vibrations, or simple test bench displacements for any reason, changing significantly our original signal as already quoted.

The straightforwardness of random sampling methods for selecting training sets turns into their major benefit. Likewise, it seems enough for the most part of real-world applications (Nalepa and M. Kawulok, 2018). However, it may be tough to deal with large and noisy datasets, because eliminating mislabeled vectors that affect the SVM performance is usually time-consuming.

We have been used random selection training for the original set (2017 data). Considering the poor results obtained for training with 2017 data and testing with 2019 data, we changed the strategy. From the previous discussion, random sampling method, and avoiding mislabeled samples, we propose: for the training, using the entire set of data acquired in 2017 plus $5 \%$ of the 2019 data.

This $5 \%$ was taken up from a time window with low noise indeed. We called that adaptative update training set. The remaining $95 \%$ were used for the test data.

As can be seen in Table 7, we achieved some improvement by means of this strategy (when compared with Table 6), but still unsatisfactory. Afterward, we adaptative update with $10 \%$ of the 2019 data. This $10 \%$ are selected in the same way as the last try (5\%). Results increased quite significantly.

We chose to show only the static unbalance kind and level 1 severity because of the little current amplitude variation, which makes diagnosis tougher. For all other cases, the results are even better.

We emphasize the importance of maintaining the 2017 data training, in order to keep the record for the maintenance engineering context. 
Table 7. Static unbalance kind and level 1 severity $-5 \mathrm{~Hz}$ analyses with multiclass SVM

\begin{tabular}{llll}
\hline & Pure 2017 data training & $\begin{array}{l}2017 \text { plus 5\% from } \\
2019 \text { data training }\end{array}$ & $\begin{array}{l}2017 \text { plus } 10 \% \text { from } \\
2019 \text { data training }\end{array}$ \\
\hline Frequency & Accuracy $(\%)$ & & 95 \\
\hline relevant & 52 & 79 & 99 \\
\hline $1,5 \mathrm{f}$ & 86 & 99 & 90 \\
\hline $0,5 \mathrm{f}$ & 77 & 83 & 96 \\
\hline $0,5 \& 1,5 \mathrm{f}$ & 49 & 85 & \\
\hline
\end{tabular}

\section{Conclusion}

Rotor unbalances due to typical usage is a very common problem in industries. With a predictive diagnosis by motor monitoring conditions, we can save money with maintenance engineering and reduce downtime.

Here, we presented a strategy to rotor unbalance kind and severity identification. A laboratory test bench was built according to ISO's standards. As the industrial environment is oftentimes intricate, our choice was to use the stator current signature analysis. We employ a hall effect current transducer and a data acquisition board.

For the analysis of current signals, we use FFT-Hilbert Transform as input signals to ML techniques.

We selected three supervised learning strategies: OvO, DTC, and SVM. The rotor unbalance problem is nonlinear and multiclass, and these three algorithms deal well under these conditions. Another main characteristic of these three algorithms is the easy implementation, training, exploration of results as well availability of open source libraries.

OvO, DTC and SVM have similar results here. However, SVM showed up high sensibility to frequency bins outside the harmonics signal because they have low energy random behavior. As discussed by Hussein (2019), this source of confusion has also been found here. This is not a drawback for the OvO and DTC.

Satisfactory results are obtained for training and testing with 2017 data. However, for training with 2017 data and testing with 2019 data the classification does not work so well. From 2017 to 2019, misalignments occurred due to ambient temperature range, building vibrations, or simple laboratory test bench displacements for any reason can explain the poor results. Such occurrences are much more frequent in an industrial environment.

To overtake these issues, we proposed the data set adaptative update. We take $10 \%$ of data from 2019 and put it into 2017 data training. This $10 \%$ is a time window with low noise. The classification rate goes to over $99 \%$. We agree it is decisive to maintain the original 2017 data training for the maintenance engineering framework.

Future work will focus on strategies for the insertion of more failure kinds such as lack of lubrication in the bearings. Always employing a robust hardware and software framework for industrial applications. With more information and how they are interpreted, the easier is the diagnosis.

\section{References}

Antonino-Daviu, J., \& Popaleny, P. (2018). Detection of induction motor coupling unbalanced and misalignment via advanced transient current signature analysis. XIII International Conference on Electrical Machines, 2359-2364. https://doi.org.10.1109/ICELMACH.2018.8506949

Baccarini, L. M. R. et. al. (2011). SVM practical industrial application for mechanical faults diagnostic. Expert Systems with Applications. https://doi.org.10.1016/j.eswa.2010.12.017

Chao, L. et. al. (2019). A novel reconstructed training-set SVM with roulette cooperative coevolution for financial time series classification. Expert Systems with Applications. https://doi.org.10.1016/j.eswa.2019.01.022

Chen, J. et. al. (2016). Wavelet transform based on inner product in fault diagnosis of rotating machinery: A review. Mechanical Systems and Signal Processing. https://doi.org.10.1016/j.ymssp.2015.08.023

Feng, Z., Zhang, D., \& Zuo, M. J. (2017). Adaptive mode decomposition methods and their applications in signal analysis for machinery fault diagnosis: a review with examples. IEEE Access. https://doi.org.10.1109/ACCESS.2017.2766232

Finley, W. R. et. al. (2015). Motor vibration problem: How to diagnose and correct vibration errors. IEEE Industrial Application Magazine. https://doi.org.10.1109/MIAS.2014.2345811

Global Market Insights. (2019). Electric Motors Market Size - Industry Trends Analysis Report 2017-2024, June. Retrieved from https://www.gminsights.com/industry-analysis/electric-motors-market

Hey, J. et. al. (2016). Online monitoring of electromagnetic losses in an electric motor indirectly through temperature measurement. IEEE Transactions on Energy Conversion. https://doi.org.10.1109/TEC.2016.2562029 
Hussain, S. F. (2019). A novel robust kernel for classifying high-dimensional data using SVM. Expert Systems with Applications. https://doi.org.10.1016/j.eswa.2019.04.037

Khan, S., \& Yairi, T. (2018) A review on the application of deep learning in system health management. Mechanical Systems and Signal Processing. https://doi.org.10.1016/j.ymssp.2017.11.024

Liu, Y. (2019). Python machine learning by example: implement machine learning algorithms and techniques to build intelligent systems. Packt Publishing.

Matić, D. et. al (2012). Support vector machine classifier for diagnosis in electrical machines: Application to broken bar. Expert Systems with Applications. https://doi.org.10.1016/j.eswa.2012.01.214

Matsushita, O. et al. (2017). Vibrations of rotating machinery. Springer.

Nalepa, J., \& Kawulok, M. (2018). Selecting training sets for support vector machines: a review. Artificial Intelligence Review, Springer Netherlands.

Rahman, M. M., \& Uddin, M. N. (2017). Online unbalanced rotor fault detection of an IM drive based on both time and frequency domain analyses. IEEE Transactions on Industry Applications. https://doi.org.10.1109/TIA.2017.2691736

Rigoni, M. et. al. (2017). Non-invasive monitoring system of synchronous generator using external field. Journal of Microwaves, Optoelectronics and Electromagnetic Applications. https://doi.org.10.1590/217910742017v16i1872

Scikit-learn. (2020). Machine Learning in Python. Retrieved from https://scikit-learn.org/stable/

Shrivastava, A., \& Mohanty, A. R. (2020). Identification of unbalance in a rotor-bearing system using Kalman filterbased input estimation technique. Journal of Vibration and Control. https://doi.org.10.1177/1077546319891642.

Thomson, W. T., \& Culbert, I. (2017). Current signature analysis for condition monitoring of cage induction motors: industrial application and case histories. Wiley-IEEE Press.

Toliyat, H. et. al. (2017). Electric machines: modelling, condition monitoring, and fault diagnosis. CRC Press.

Tsui, K. L. et. al. (2014). A one-vs-all class binarization strategy for bearing diagnostics of concurrent defects. KLSensors, Basel. https://doi.org.10.3390/s140101295

\section{Copyrights}

Copyright for this article is retained by the author(s), with first publication rights granted to the journal.

This is an open-access article distributed under the terms and conditions of the Creative Commons Attribution license which permits unrestricted use, distribution, and reproduction in any medium, provided the original work is properly cited. 